Musées, Patrimoine et Culture scientifiques et techniques

$195 \mid 2021$

mai-juin 2021

\title{
Le musée, créateur de liens. Collecte d'Émotions patrimoniales au Laténium
}

Géraldine Delley

\section{OpenEdition \\ Journals}

Édition électronique

URL : https://journals.openedition.org/ocim/4315

DOI : $10.4000 /$ ocim.4315

ISSN : 2108-646X

Éditeur

OCIM

Édition imprimée

Date de publication : 1 mai 2021

Pagination : 10-15

ISSN : 0994-1908

Référence électronique

Géraldine Delley, «Le musée, créateur de liens. Collecte d'Émotions patrimoniales au Laténium », La Lettre de I'OCIM [En ligne], 195 | 2021, mis en ligne le 01 mai 2021, consulté le 13 juin 2022. URL : http://journals.openedition.org/ocim/4315 ; DOI : https://doi.org/10.4000/ocim.4315

Ce document a été généré automatiquement le 13 juin 2022.

Tous droits réservés 


\title{
Le musée, créateur de liens. Collecte d'Émotions patrimoniales au Laténium
}

\author{
Géraldine Delley
}

Des vestiges de fortifications de la Seconde guerre mondiale à Cudrefin (1963). Collection particulière.

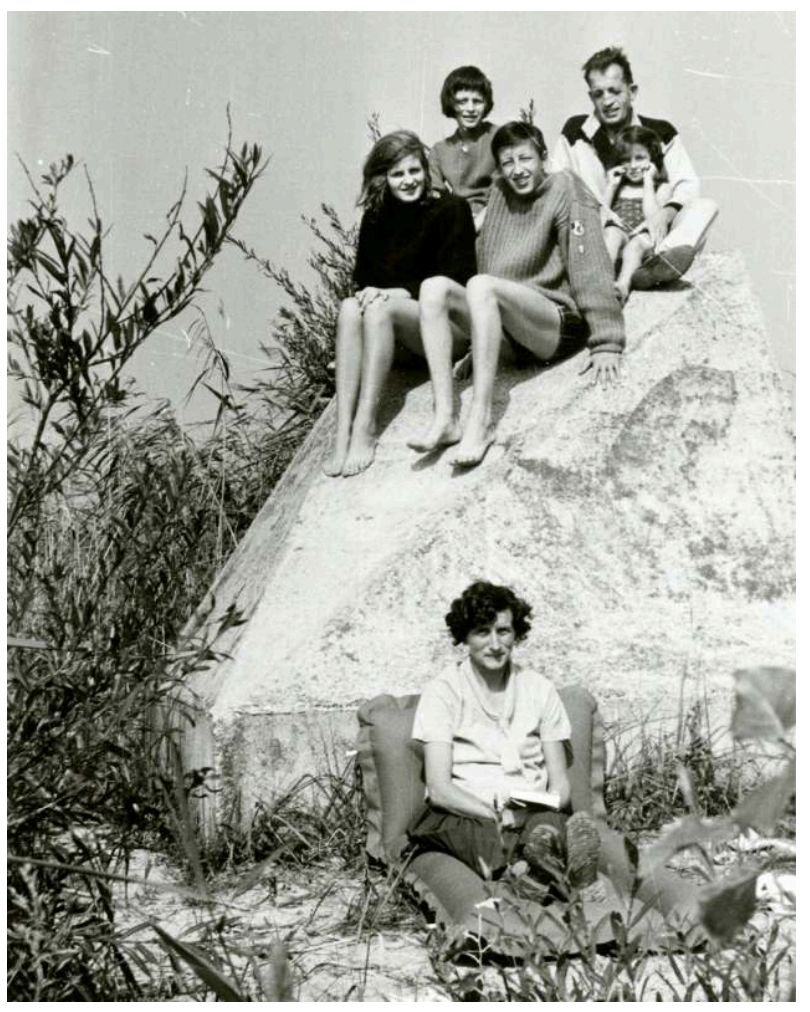

(c) DR

Afin d'accéder à des formes d'appropriations du patrimoine situées hors du champ scientifique, nous avons lancé une collecte de photographies et de témoignages qui ont formé le cœur de l'exposition. Trois autres parties mettaient en scène des objets et des 
matériaux audiovisuels dans la perspective d'inscrire les résultats de notre collecte dans un contexte plus vaste ${ }^{1}$. L'exposition prenait place dans un appartement en ruines, lieu par excellence où se trament nos vies personnelles, scénographié par Elissa Bier. Au fil de leur exploration, les visiteurs découvraient des traces - images, objets, récits et films - qui témoignaient, à la manière des vestiges étudiés par les archéologues, d'un intermédiaire entre nos vies présentes et un passé qui se transforme.

\section{La photographie de famille comme archive}

2 Dans la littérature scientifique qui traite de photographie vernaculaire, les auteurs s'entendent pour considérer que la photographie privée est une source d'information précieuse pour les sciences historiques et les sciences humaines en général. Toutefois, ce type d'archive est réputé difficile à analyser. Ce scepticisme s'explique par l'incertitude qui plane sur ces images prises souvent dans des cadres informels et qui se destinent à des groupes restreints, généralement assimilés au cercle familial ou des amis ${ }^{1}$.

3 En raison de leur fonction privée, ces clichés supposent en effet des explications verbales qui font souvent défaut. Souvent impossible à situer et donc à analyser, leur usage comme matériau de recherche est souvent délicat ${ }^{2}$.

Notre collecte (voir encadré ci-après) nous a permis d'anticiper ces problèmes d'interprétation, puisque les photographies que nous avons reçues étaient accompagnées de récits recueillis par écrit ou par le biais d'entretiens semi-dirigés conduits avec leurs donateurs ${ }^{3}$.

\section{Une collecte pérennisée}

La collecte Montre-moi ton patrimoine a pris place entre mai et novembre 2018. Elle a rassemblé des clichés montrant des gens posant devant des monuments, ruines ou vestiges se trouvant en Suisse. Nous voulions ainsi questionner les formes d'attachements ordinaires à un patrimoine local, perçu comme peu spectaculaire voire banal ${ }^{4}$. Nous voulions éviter que les donateurs aillent intentionnellement se photographier ou se faire photographier devant des vestiges pour les besoins de la collecte. Les matériaux qui nous intéressaient devaient au contraire relever d'une pratique préexistante révélée par les archives et les albums de famille papier ou numériques. La collecte a permis de réunir plus de 400 clichés pris entre 1886 et 2018, la majorité se situant dans la tranche 1950-1970, moment d'apogée de l'album photographique ${ }^{5}$. Plusieurs institutions hébergeant des fonds photographiques vernaculaires ont également pris part à notre collecte. Mais ces images restaient pour la plupart muettes, à la différence des clichés des donateurs et donatrices avec lesquels nous avons pu nous entretenir. Enfin, il était important qu'à l'issue de l'exposition, les images et témoignages collectés restent accessibles au plus grand nombre. Ils sont visibles sur la plateforme participative :

https://notrehistoire.ch/@emotions 
Vue de l'exposition Émotions patrimoniales. Scénographie E. Bier.

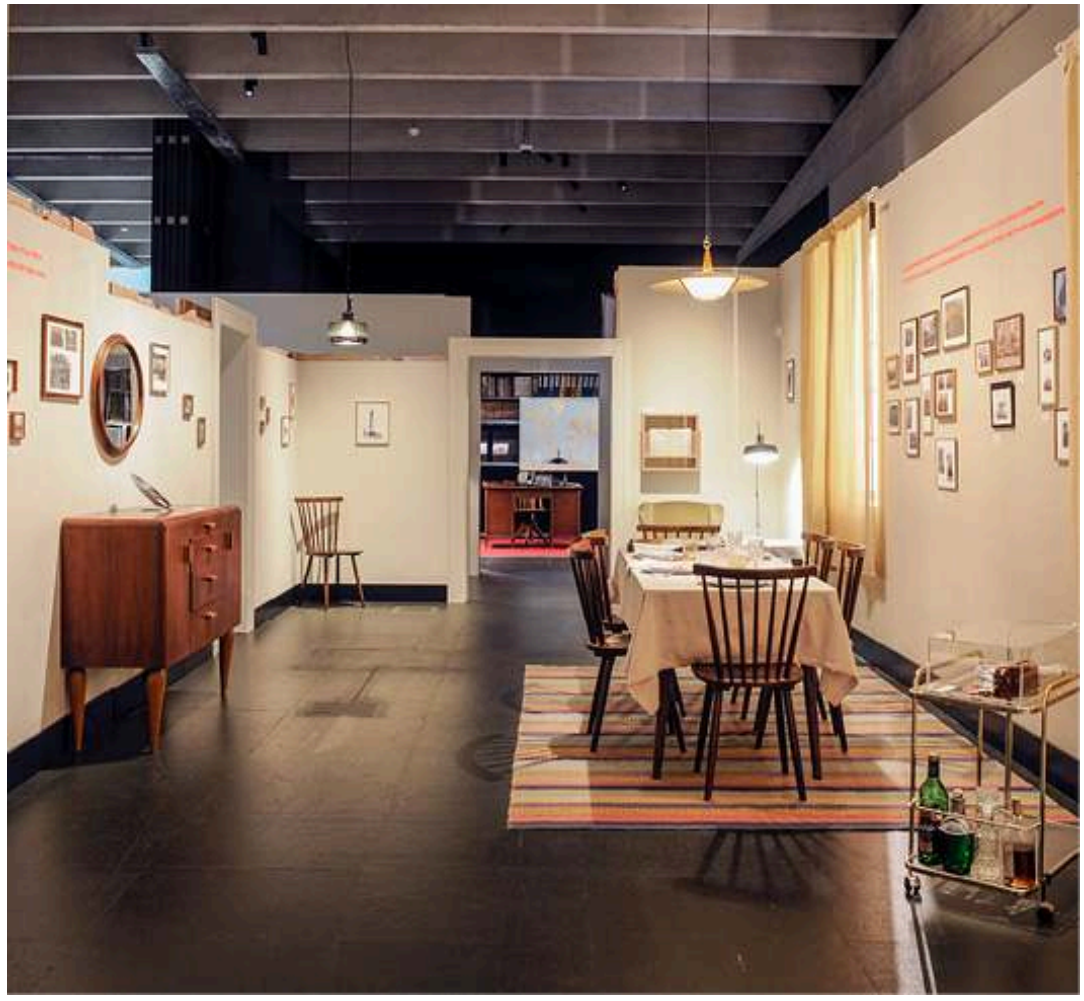

(c) M. Juillard/Laténium

\section{Les images, supports de récits}

5 À l'issue de la collecte, nous disposions donc de deux types de matériaux - images et récits - qui nous ont permis de construire le propos de l'exposition. Les clichés collectés constituaient des fragments de temps décontextualisés, des sortes de hors cadre, qu'il s'agissait de recontextualiser grâce aux entretiens menés avec les donateurs.

6 Or c'est par la médiation de l'image que la reconstruction mémorielle a pu se faire. En décrivant les photographies, les donateurs parvenaient à situer l'année où la photographie avait été prise, à énumérer les personnes qui se trouvaient sur l'image. D'autres détails tels que le contexte dans lequel la photographie avait été réalisée, des anecdotes liées à l'instant immortalisé par l'image ou l'attachement que les donateurs avaient pour l'endroit photographié ressortaient également dans le discours des personnes interrogées. Ces témoignages permettaient au final d'exprimer ce que l'image seule n'exprimait pas. Pour autant, ils ne se subordonnaient pas à l'image, mais venaient la compléter à la manière des écrits qui accompagnent certains clichés dans la littérature de voyage ${ }^{6}$.

7 Notre démarche nous a conduits à nous intéresser à deux instants précis dans l'histoire de ces photographies. Il s'agissait d'une part du moment où les photographies ont été prises, avec les motifs qui ont conduit à leur réalisation. Ces moments, qui remontaient parfois loin dans le temps, faisaient l'objet d'une reconstruction a posteriori par le donateur qui se fondait éventuellement sur des récits rapportés par d'autres membres de sa famille, en particulier lorsque lui-même n'était pas présent sur la photographie. D'autre part, il s'agissait de questionner les effets produits par ces photographies dans 
le présent, autrement dit sur les donateurs, au moment où nous leur demandions de les commenter, puis sur les visiteurs de l'exposition, pour qui ces lieux pouvaient également être chargés de souvenirs et d'émotions. Ainsi, davantage que d'interroger la pratique qui consiste à se photographier devant des traces matérialisées du passé, il s'agissait d'interroger les formes de médiations produites par ces images singulières dans le présent.

Lors de rencontres familiales, les photographies prises devant des monuments ou dans des lieux historiques ont enregistré la mémoire de l'endroit où l'événement s'est déroulé. Neuchâtel (date non précisée) est représentée par sa collégiale et le village de Thielle (1945) est rendu explicite par son château. Collection particulière.

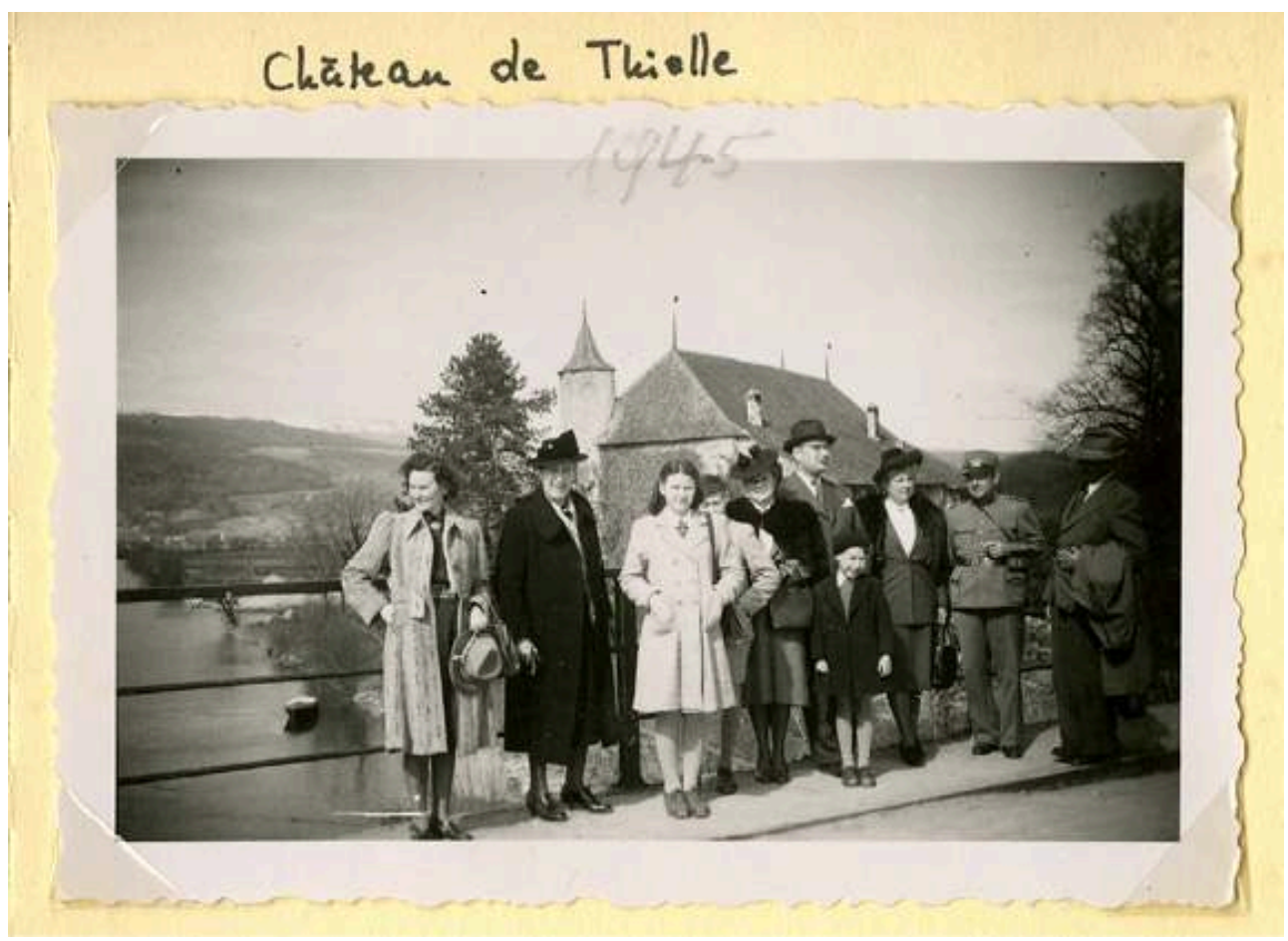


Entre les menhirs de Corcelles-près-Concise (1992). Collection particulière.

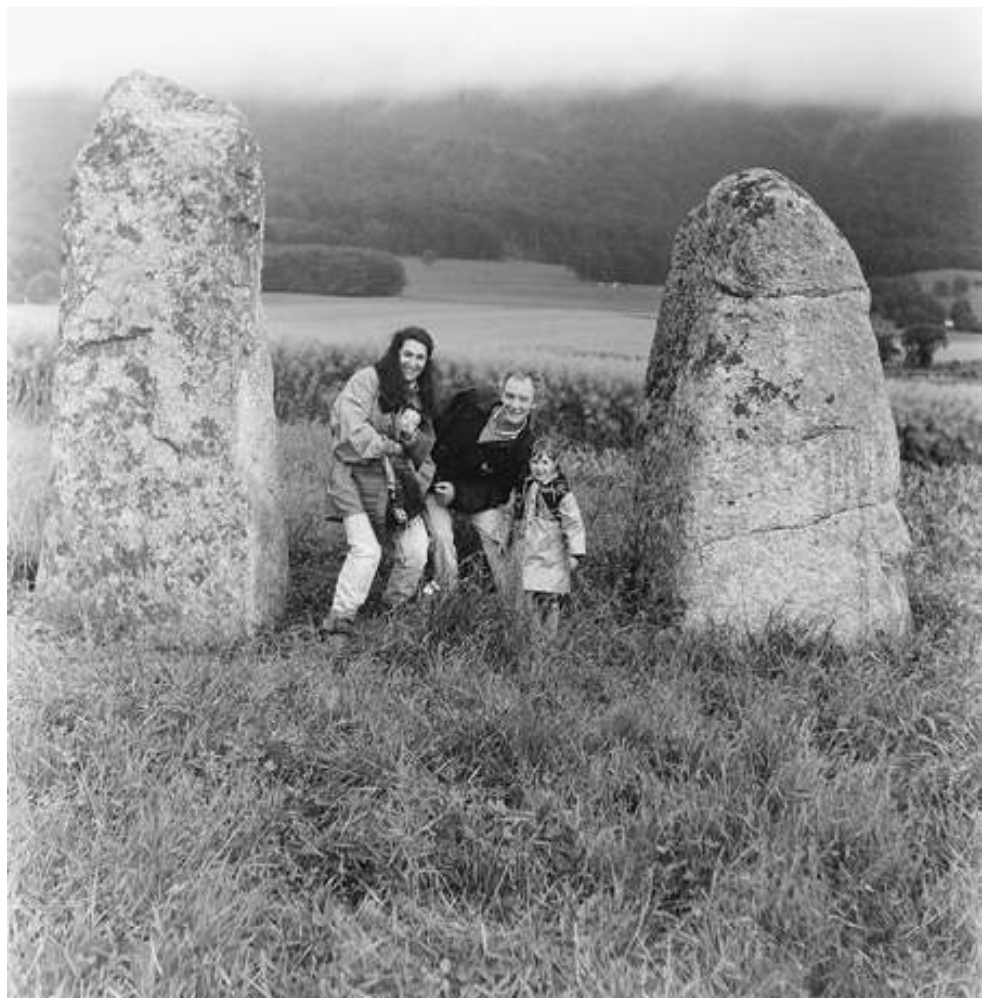

(C) DR

Des vestiges de fortifications de la Seconde guerre mondiale à Cudrefin (1963). Collection particulière.

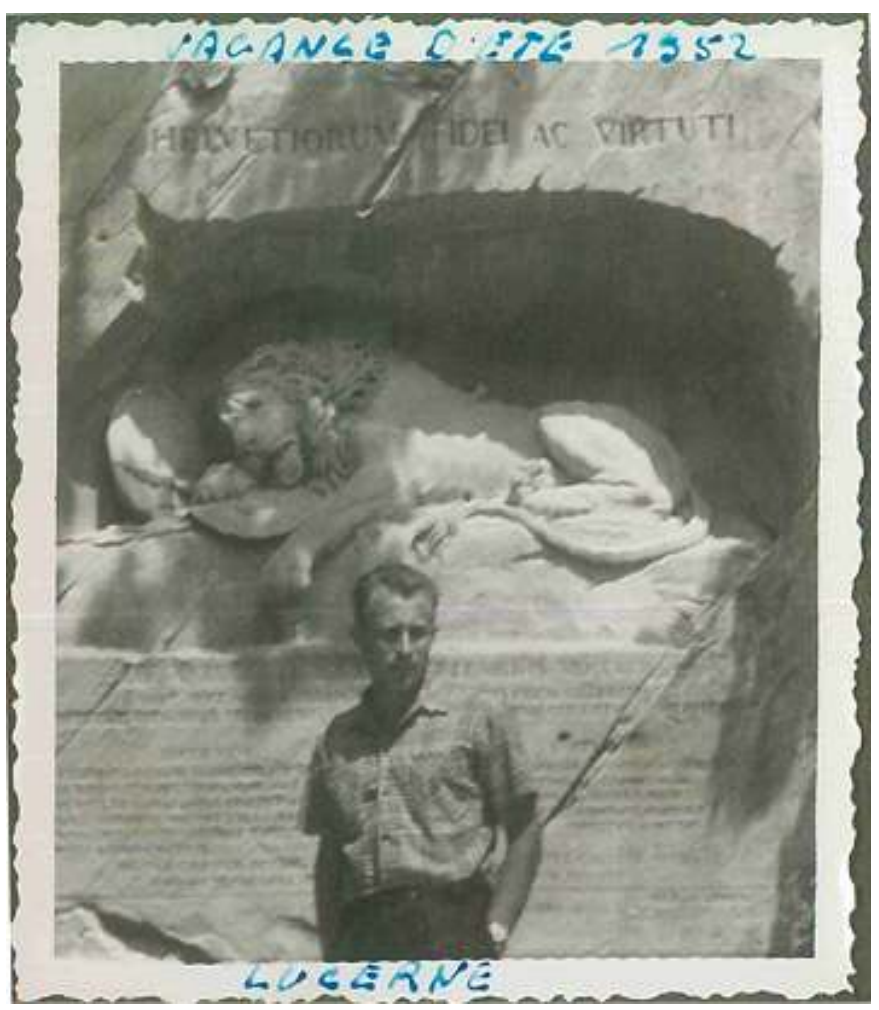

(C) DR 
Des vestiges de fortifications de la Seconde guerre mondiale à Cudrefin (1963). Collection particulière.

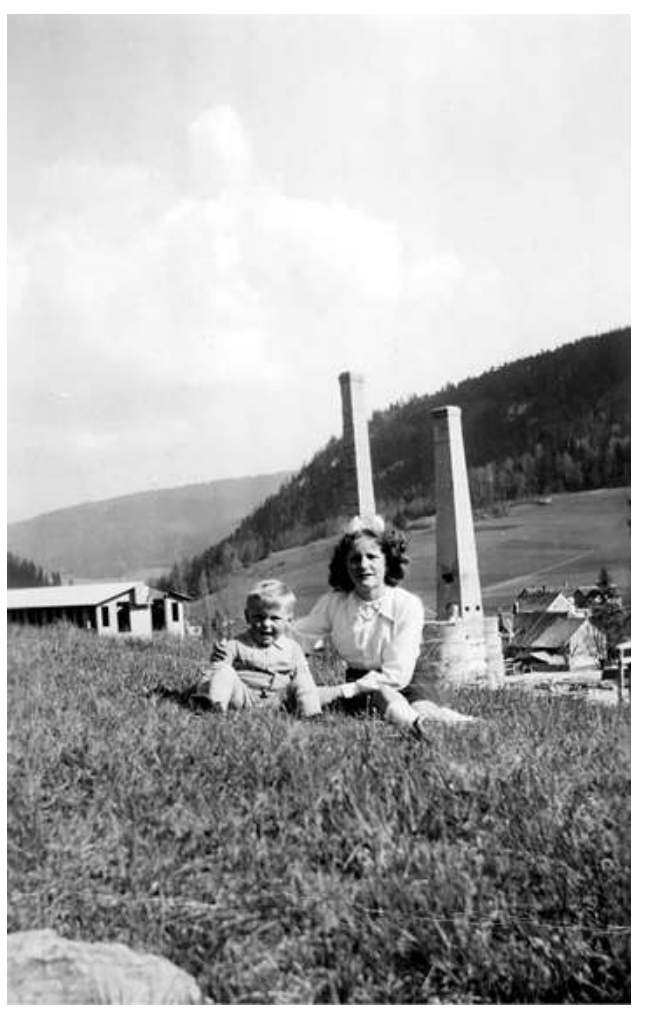

(c) DR

\section{Transformer l'ordinaire en quelque chose de remarquable}

8 En lançant notre collecte, le cadre chronologique fixé était d'abord compris entre le Paléolithique et le Moyen Âge. De nombreux donateurs n'ont cependant pas respecté ces limites qui devaient leur paraître peu représentatives de l'idée qu'ils se faisaient du patrimoine. Ainsi, un nombre important de clichés reçus rendaient compte de monuments datant du XIX siècle et allant même jusqu'au début du XXI ${ }^{e}$ siècle. Nous les avons retenus pour aborder dans notre exposition la question de ce qui «fait patrimoine » auprès des gens.

De par la nature du patrimoine photographié, des vestiges et des monuments dont la portée était volontairement locale, les témoignages qui nous sont parvenus révèlent des gestes inconscients et des détails marginaux (Ginzburg, 2010 [1979]) dans ce qui se joue autour des relations intimes tissées entre ces lieux de patrimoine et ceux qui les ont fréquentés. C'est parfois à partir d'impressions vécues qui n'étaient pas précisément liées au lieu visité, mais qui relevaient de ce qui avait entouré la visite que les participants décrivaient ces photographies.

10 Ainsi, en reconstruisant les souvenirs qui lui restaient d'un après-midi ensoleillé de 1953 passé en famille sur le site romain d'Avenches, un donateur nous expliquait à quel point les baskets qu'il avait aux pieds ce jour-là avaient changé sa vie. Il se souvenait également que la casquette qu'il portait sur la photographie lui avait été prêtée par le gardien du musée. Il en était très fier et avait décidé de poser, accompagné de sa mère, 
devant les ruines de l'amphithéâtre que l'on voit depuis l'une des salles du musée. Cette visite était également synonyme de voyage en voiture à une époque où ce mode de transport n'était pas répandu. Ses parents n'ayant pas de véhicule, c'était leur voisine qui avait conduit toute sa famille à Avenches. Il se souvenait alors de nombreux détails du voyage qui l'avait amené à quitter La Chaux-de-Fonds où il vivait.

Une autre participante nous expliquait que la photographie qu'elle nous avait transmise avait été prise alors qu'elle était en vacances au Tessin avec sa mère. Son père, qui était resté travailler à Neuchâtel, les avait rejointes et avait insisté pour prendre une photographie d'elles devant l'église baroque de la Madonna del Sasso audessus de Locarno. Comme toujours, nous racontait la donatrice, ça a pris du temps pour faire la photographie. C'était très inconfortable car elle avait le soleil dans les yeux, d'où la grimace sur le cliché.

Avenches, Tour de la Tornallaz (2018). Collection particulière.

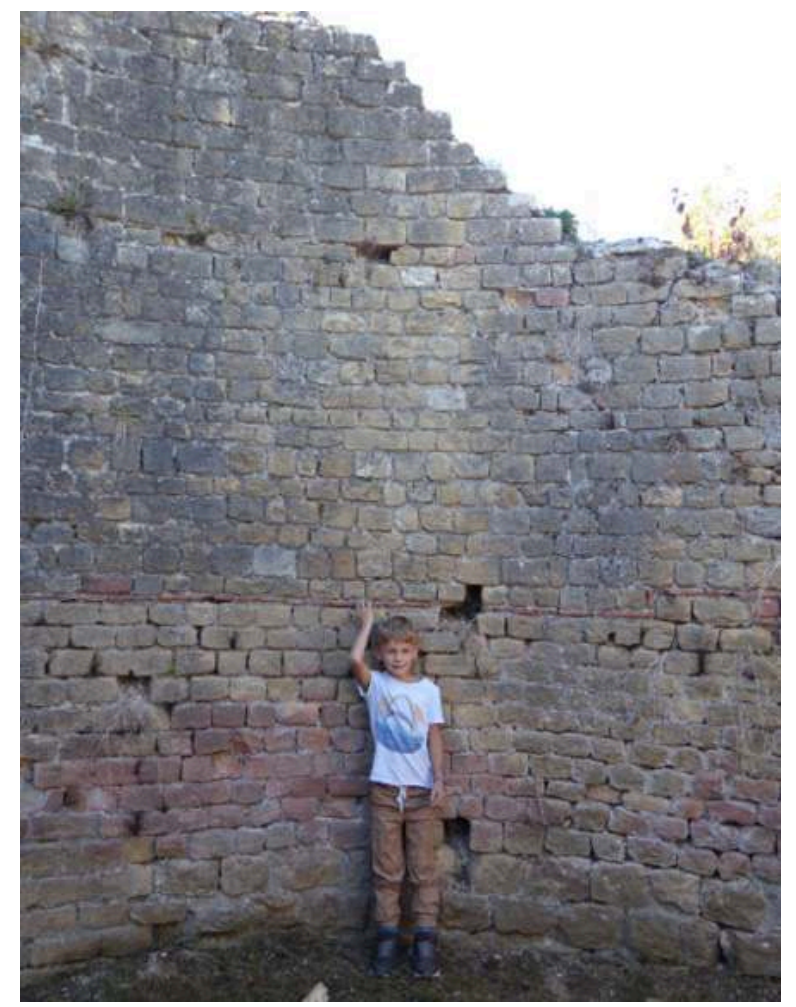

(c) DR

12 Le contraste entre ces détails relevant à la fois de la précision et de la banalité renvoie à quelque chose d'étonnant: le patrimoine semble donner un caractère peu commun à des fragments de vie qu'on aurait peut-être oubliés si ces photographies avaient été prises ailleurs. Le patrimoine étant constitutif de références communes qui s'inscrivent dans une mémoire visuelle collective, il tend à transformer l'ordinaire en quelque chose de remarquable et qui est digne d'être enregistré par la mémoire ${ }^{7}$. Il en ressort que c'est parce qu'il y a du patrimoine sur ces photographies que l'on se souvient du moment photographié. 


\section{Ritualiser les étapes de l'existence}

13 Parmi les clichés reçus, nombreuses sont ceux qui ont été pris lors de mariages et de baptêmes, où les gens posent en couple ou en groupe devant des monuments. Le patrimoine semble ici jouer un rôle d'amplificateur de moments solennels. On se photographie dans des lieux de patrimoine lors de moments officiels ou encore, comme dans les deux exemples qui suivent, pour marquer la tradition de rencontres familiales.

Un exemple particulièrement intéressant est celui d'une famille posant entre deux menhirs à Corcelles-près-Concise en 1992. Son donateur nous a expliqué que cette image est un instantané de sa famille à un moment précis qui est celui de la naissance de leur dernier enfant. "[...] C'est une réelle émotion, une intension précise, on se rend là et nulle part ailleurs, les menhirs c'est pérenne, ça vient du passé ». Ces vestiges constituent des repères, tels des points fixes dans le paysage. C'est dans cette temporalité qui le dépasse et qui lui survit que ce donateur a choisi de venir inscrire la trace d'un moment précis de sa biographie familiale.

\section{Des repères dans les géographies intimes}

Parmi les témoignages que nous avons collectés, le patrimoine constitue souvent un but de balade. Dans ces itinéraires répétés, c'est l'expérience de retourner dans des lieux connus que l'on affectionne particulièrement qui est recherchée. Les formes d'appropriation de ces vestiges peuvent être multiples: espaces de jeux, lieux de méditation, repères, etc. Pour plusieurs personnes interrogées, ces vestiges sont constitutifs de leur géographie intime au sens où ces lieux sont devenus un peu les leurs. Ils constituent des repères, au sens propre, dans les biographies personnelles, comme l'indiquent les témoignages suivants : «En allant prendre le goûter à la [tour de la] Tornallaz, nous avons découvert cette ligne rouge dans le mur et décidé que nous reviendrons régulièrement mesurer Jamie, et quand il y reviendra, adulte, il se rappellera de sa première mesure ». Dans cet exemple, la ligne rouge en question fait partie du monument. Or c'est la durabilité de ce dernier qui confère à la marque colorée son rôle de jalon. Un autre cas est celui d'une donatrice qui nous expliquait qu'elle avait pour habitude de photographier ses amis français venus lui rendre visite devant des menhirs. Situés près du lieu où elle habite, ces pierres dressées constituent des repères dans son parcours personnel. Suissesse d'adoption, elle connaissait les menhirs bretons mais ignorait qu'en Suisse on en trouvait également. Ces monuments constituent en quelque sorte des traits d'union entre son pays d'origine, la France, et l'endroit où elle vit aujourd'hui, la Suisse.

\section{La photographie comme trace de la trace}

Les clichés que nous avons collectés constituent des preuves matérielles de morceaux de vie voués à disparaître où les monuments et les vestiges constituent des repères stables. La photographie de soi devant ces traces du passé répond alors «à ce besoin fondamental de l'homme d'arrêter la marche du temps [...] " $\|^{8}$. Mais ces photographies ont également un pouvoir d'attestation au sens où elles ont une valeur de preuve de ce qui a existé et qui est rapporté par l'image. Dans certains témoignages que nous avons collectés, la photographie commentée est devenue une trace de la trace. Comme l'a 
montré Marta Caraion qui s'est intéressée au rapport aux ruines dans la photographie et la littérature de voyage, l'image permet ici de faire une histoire de soi et une histoire du monde, en collectionnant le présent avant qu'il ne devienne ruine ou qu'il ne disparaisse. Ainsi, si le patrimoine ou les vestiges sont des ruines en devenir, derrière la photographie, lorsqu'elle est commentée a posteriori, c'est le récit de sa propre vie en tant que totalité passée qui est aussi invoqué 9 .

\section{BIBLIOGRAPHIE}

Caraion M. Pour fixer la trace. Genève : Droz, 2003.

Chéroux C. Vernaculaires : essais d'histoire de la photographie. Cherbourg-Octeville : Le Point du Jour, 2013.

Delley G. Émotions patrimoniales. Catalogue numérique de l'exposition/Digitaler Katalog der Sonderausstellung. Hauterive : Laténium, 2020 :

https://latenium.ch/wp-content/uploads/2020/04/latenium-emotions-PDF.pdf

Dunning E. Lieux de mémoire et photographie amateur : les sites archéologiques révélateurs d'émotions patrimoniales, in Debary O. et Bonnefoit R. (dir.) Postérités des Lieux de mémoire. Entretiens avec Pierre Nora. Neuchâtel : Éditions Alphil, à paraître.

Fabre D. (dir.) Émotions patrimoniales. Paris : Éditions de la Maison des sciences de l'Homme, 2013. Isnart C. Les patrimonialisations ordinaires. Essai d'images ethnographiées, ethnographiques.org, $\mathrm{n}^{\circ} 24$, juillet 2012 :

www.ethnographiques.org/2012/Isnart

Ginzburg C. Traces. Racines d'un paradigme indiciaire, in Mythes, emblèmes, traces : morphologie et histoire. Lagrasse : Verdier, 2010 [1979], pp. 139-287.

Pagenstecher C., Private Fotoalben als historische Quelle, Zeithistorische Forschungen/Studies in Contemporary History, Heft 3, 2009, pp. 449-463 :

https://doi.org/10.14765/zzf.dok-1803

Rebaud P. Concessions à perpétuité. Paris : La vie est belle films, 1997, 52 min.

Rautenberg M. La rupture patrimoniale. Paris : À la croisée, 2003.

Sandbye M. Looking at the family photo album: a resumed theoretical discussion of why and how, Journal of Aesthetics \& Culture, vol. 6, n 1, 2014, pp. 1-17.

Sontag S. Sur la photographie. Paris : C. Bourgois, 2008 [1979].

\section{NOTES}

1. Pagenstecher, 2009 ; Sandbye, 2014 ; Chéroux, 2013.

2. Le soin accordé à ces fonds photographiques reste très circonstanciel. Signalons qu'une table ronde a été consacrée au sujet en mai 2015. Organisée par Fabrice Denise, elle réunissait plusieurs 
acteurs engagés dans la valorisation de fonds vernaculaires : Jean-Pierre Moulières, Maïté Labat, Luce Lebart, Anne-Marie Garat et Sam Stourdzé. Cette rencontre prenait place dans le cadre de l'exposition temporaire J'aimerais tant voir Syracuse au musée départemental Arles antique.

3. Les entretiens ont été conduits par Ellinor Dunning qui a tiré de son expérience un article sur le lien entre les clichés collectés et la notion de lieux de mémoire (voir Dunning, à paraître).

4. Notre démarche venait ainsi compléter les enquêtes menées par le Lahic (Fabre dir. 2013) qui rendent compte d'investissements émotionnels suscités par un tel patrimoine local. Voir également Isnart, 2009 et, concernant l'archéologie en particulier, le documentaire de Patrick Rebaud (1997) dont des extraits étaient présentés dans l'exposition.

5. Pagenstecher, 2009, p. 452.

6. Voir à ce propos Caraion, 2003, pp. 98-99.

7. Cela n'est sans doute pas une spécificité du patrimoine historique et archéologique, le même phénomène aurait sans doute lieu avec une photographie du patrimoine naturel, dont on sait qu'il est également générateur d'émotions. Sur les enjeux très actuels liant patrimoine naturel et mémoire collective, voir notamment l'abondante littérature produite par l'Unesco et le Conseil de l'Europe autour des concepts de «natural and cultural heritage».

8. Caraion 2003, p. 87. Marta Caraion s'inspire ici des propos de Ernest Lacan dans «De la photographie et de ses diverses applications appliquées aux beaux-arts et aux sciences ", La Lumière, 20 janvier 1855, p. 29.

9. Ibid. p. 271 et suivantes.

\section{RÉSUMÉS}

Une collecte participative a conduit à interroger le sens que vestiges et monuments revêtent aux yeux du public, les émotions qu'ils suscitent et les récits qu'ils génèrent. Entre mai 2019 et janvier 2020, l'exposition Émotions patrimoniales au Laténium explorait les formes d'attachement que la population suisse éprouve pour le patrimoine archéologique et historique.

\section{INDEX}

Mots-clés : dispositif participatif, patrimoine et/ou collection archéologiques, monument et site archéologique

\section{AUTEUR}

\section{GÉRALDINE DELLEY}

Directrice adjointe du Laténium à Neuchâtel (Suisse).

geraldine.delley@ne.ch 